\title{
Comparative Evaluation of Coronally Advanced Flap with Platelet-rich Fibrin vs Amniotic Membrane for the Treatment of Gingival Recession
}

\author{
Abhishek Gautam
}

\begin{abstract}
Background: The purpose of this clinical trial was to compare the outcome of gingival recession technique using the coronally advanced flap (CAF) with platelet-rich fibrin (PRF) and amniotic membrane.
\end{abstract}

Materials and methods: About 15 patients with bilateral Miller class I buccal gingival recessions $(4-5 \mathrm{~mm}$ ) in maxillary and mandibular canines were selected. The recessions were randomly assigned to receive either the CAF with PRF or the CAF with amniotic membrane. Recession height $(\mathrm{RH})$, recession width (RW), width of keratinized tissue (WKT), thickness of keratinized tissue (TKT), probing depth (PD), and clinical attachment level (CAL) were measured at baseline as well as 6 months postsurgery. Patient satisfaction with esthetics, root sensitivity, and postoperative pain was also evaluated.

Results: The average percentages of root coverage for CAF with PRF and CAF with amniotic membrane were $92.95 \%$ and $97.40 \%(p<0.05)$ respectively, and the complete root coverage observed was $56.94 \%$ and $76.47 \%$ respectively. The CAF with amniotic membrane showed a statistically significant increase in TKT $(p<0.05)$. There were no significant differences between the two groups with regard to $\mathrm{RH}, \mathrm{RW}, \mathrm{WKT}, \mathrm{PD}$, and CAL. The esthetic condition after both treatments was considered satisfactory by the patients.

Conclusion: The outcome of this study reflects that CAF with PRF and amniotic membrane can be successfully used to treat class I gingival recession.

Keywords: Amniotic membrane, Coronally advanced flap, Gingival recession, Platelet-rich fibrin.

How to cite this article: Gautam A. Comparative Evaluation of Coronally Advanced Flap with Platelet-rich Fibrin vs Amniotic Membrane for the Treatment of Gingival Recession. J Health Sci Res 2017;8(1):31-37.

\section{Source of support: Nil}

Conflict of interest: None

\section{INTRODUCTION}

Gingival recession designates the oral exposure of root surface due to the displacement of gingival margin apical

Private Practitioner

Abha Prakash Dental Clinic, Bodh Gaya, Bihar, India

Corresponding Author: Abhishek Gautam, Private Practitioner Abha Prakash Dental Clinic, Bodh Gaya, Bihar, India, Phone: +91-9506305424, e-mail: dr.abhi90@gmail.com to the cementoenamel junction (CEJ). ${ }^{1}$ Vigorous tooth brushing causes tissue trauma, which is considered to be one of the predominant causative factors for development of recession, particularly in young adults; however, the other factors, such as alveolar bone dehiscence, high muscle attachment or frenum pull, plaque and calculus, and iatrogenic factors are also responsible for the same. ${ }^{2}$ The laterally positioned flap technique, free gingival graft technique, connective tissue graft techniques, ${ }^{3,4}$ free gingival graft/coronally positioned flap technique, and acellular dermal matrix technique have been used to enhance esthetics and hypersensitivity of exposed root surface. However, in comparison with the abovediscussed techniques, the coronally advanced flap (CAF) introduced by Norberg (1926) is an easy and more predictable esthetic surgical procedure for treating recession, and it also eliminates the need for second surgical site.

\section{PLATELET-RICH FIBRIN}

Choukroun et $\mathrm{al}^{5}$ of France was the first who described platelet-rich fibrin (PRF) is a second-generation platelet concentrate and suggested it as an autologous leukocyte and PRF (L-PRF) biomaterial. ${ }^{6}$ Unlike other platelet concentrates, PRF requires neither anticoagulants nor bovine thrombin (nor any other gelling agent). It results from a natural and progressive polymerization occurring during centrifugation $^{7}$ which accumulate platelets, leukocytes, and their released cytokines in a three-dimensional (3D) fibrin network. ${ }^{6}$

\section{AMNIOTIC MEMBRANE}

The amnion is a membrane, building the amniotic sac that surrounds and protects an embryo. It is a tissue of fetal origin, which was used for the transplantation of skin in 1910, and is composed of three major layers: A single epithelial layer, a thick basement membrane, and an avascular mesenchyme. ${ }^{8,9}$ It contains no nerves, muscles, or lymphatics and can be easily separated from the underlying chorion. With improvements in the processing and storage technologies, amniotic membrane has found application in various fields of medicine, including management of burns, reconstruction of the oral cavity, bladder, vagina, tympanoplasty, arthroplasty, and so on. ${ }^{10}$ 
Nowadays, this multipurpose tissue has been found very useful in the field of periodontics. Velez et $\mathrm{al}^{11}$ analyzed the effects of cryopreserved amniotic membrane on periodontal soft tissue healing and observed that it was effective in helping in wound healing, epithelization, facilitated migration, and reinforced adhesion. Another report by Gurinsky ${ }^{12}$ demonstrated that the processed allograft amnion may provide an effective alternative to autograft tissue in the treatment of recession defects. Here, we present a study where an amnion membrane has been used for the management of gingival recession.

\section{MATERIALS AND METHODS}

In the present study, 15 patients ( 10 females and 5 males) aged between 21 and 52 years were included in study. All patients were nonsmokers who were found periodontally and systemically healthy with neither contraindication for periodontal surgery nor medicated to interfere with periodontal tissue health or healing. For the treatment, following criteria have been used because of presence of Miller class I gingival recessions (4-5 $\mathrm{mm}$ ) in maxillary and mandibular canines; probing depth (PD) $<3 \mathrm{~mm}$ without bleeding on probing; width of keratinized tissue (WKT) $2 \mathrm{~mm}$ and tooth vitality and absence of caries or restorations in the areas to be treated. The subjects were selected from patients referred for dental treatment at Chandra Dental College, Barabanki, Uttar Pradesh, India, from whom consent was taken.

\section{Clinical Parameters}

All measurements were recorded at baseline and after surgery (6 months), which were quantified with a caliper of 0.01-mm resolution. The baseline measurements were recorded 28 days after the initial therapy. Measurements were done from the incisal border of the CEJ (reference $\mathrm{CEJ}$ ), to the mucogingival junction (reference MGJ) and to

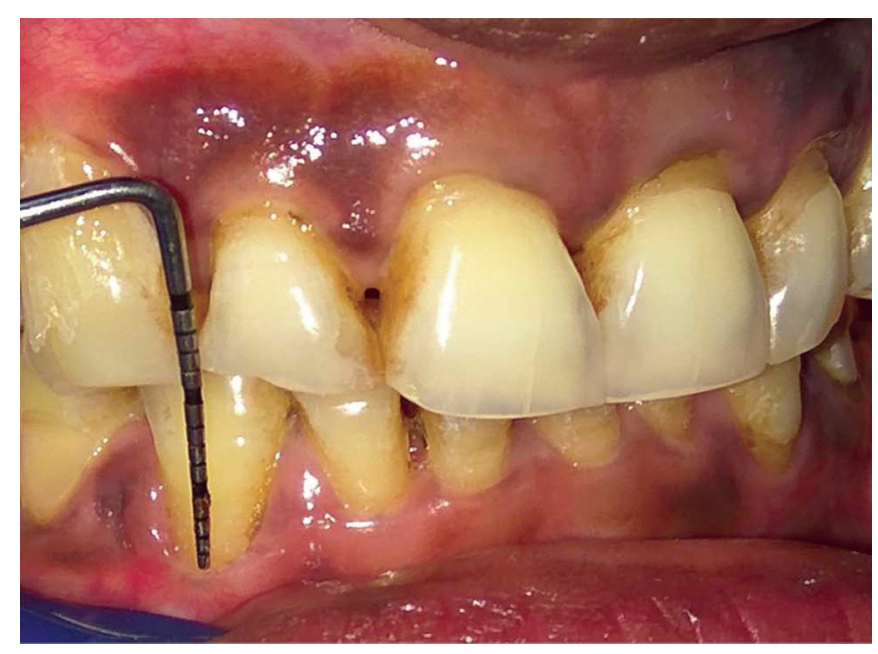

Fig. 1: Miller class II gingival recession in the lower right canine the gingival margin (reference GM) using an endodontic finger spreader attached to a rubber stopper. The following clinical parameters were assessed:

- Recession height $(\mathrm{RH})$ measured as the distance from the CEJ to the GM, calculated as reference GM ref $\mathrm{CEJ}$;

- Width of keratinized tissue measured as the distance between the most apical point of the GM and the MGJ, calculated as ref MGJ - ref GM;

- Recession width (RW), measured from one border of the recession to another at the CEJ;

- Probing depth, measured as the distance from the GM to the bottom of the gingival sulcus;

- Clinical attachment level (CAL), calculated as RH + PD; and

- Thickness of the keratinized tissue (TKT). The TKT was assessed at two times: TKT1, $2 \mathrm{~mm}$ apical to the GM before surgery and TKT2, $2 \mathrm{~mm}$ apical to the GM after surgery. After carefully removing the spreader, penetration depth was measured with a caliper of $0.01-\mathrm{mm}$ resolution. The identification of the MGJ was facilitated by staining the tissues with iodine solution. The RW and PD were measured.

\section{Surgical Procedure}

\section{Coronally Advanced Flap with Platelet-rich Fibrin}

The operative site was anesthetized using $2 \%$ xylocaine with adrenaline (1:200,000). A CAF technique was performed at the surgical site in relation to 13 (Fig. 1) by making two horizontal incisions with respect to the distal and mesial interdental papillae of 13 , followed by a crevicular incision, two vertical releasing incisions at the mesial and distal aspects of 13. A full thickness flap followed by a partial thickness one was reflected (Fig. 2). A horizontal releasing incision was made in the periosteum, at the base of the flap, to facilitate tension-free coronal

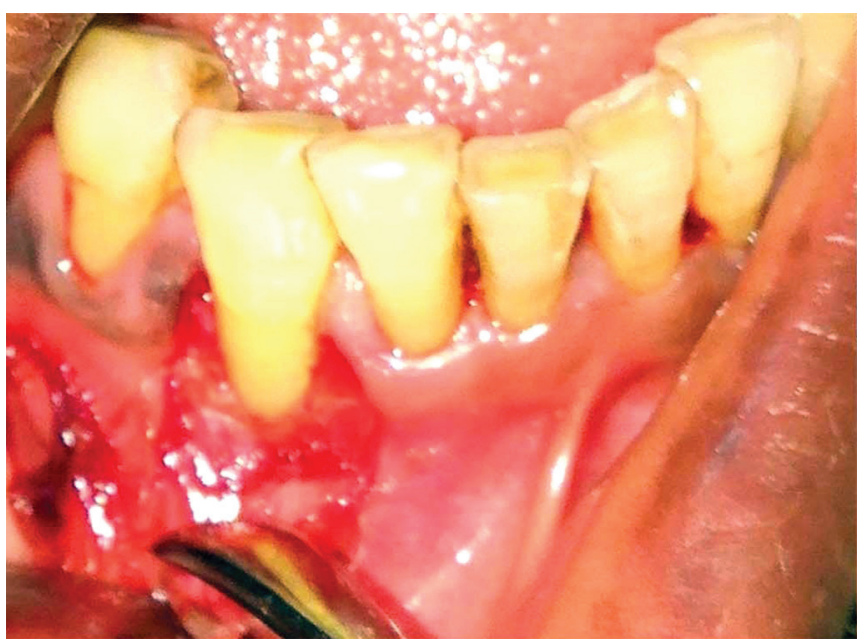

Fig. 2: Elevation of periosteal flap 


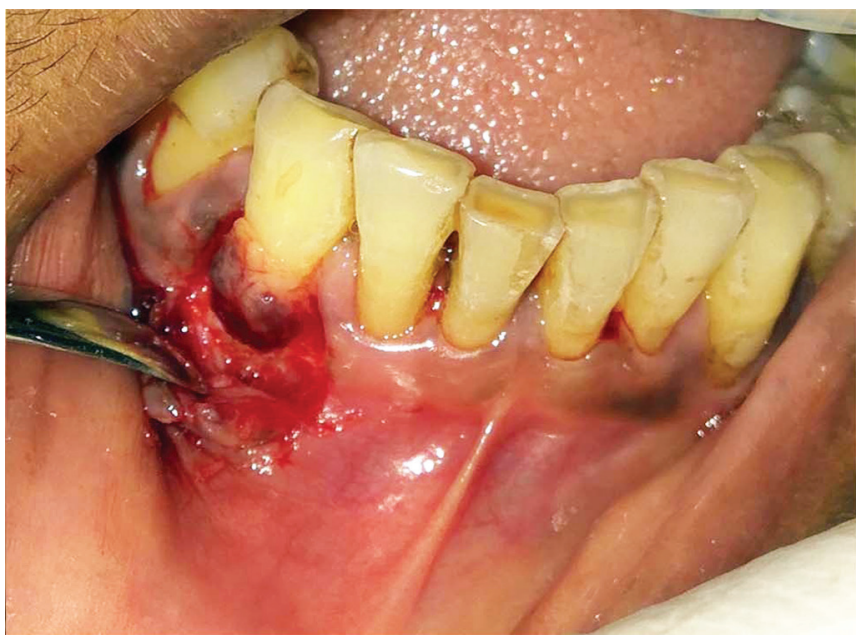

Fig. 3: Placement of PRF membrane over the exposed root surface

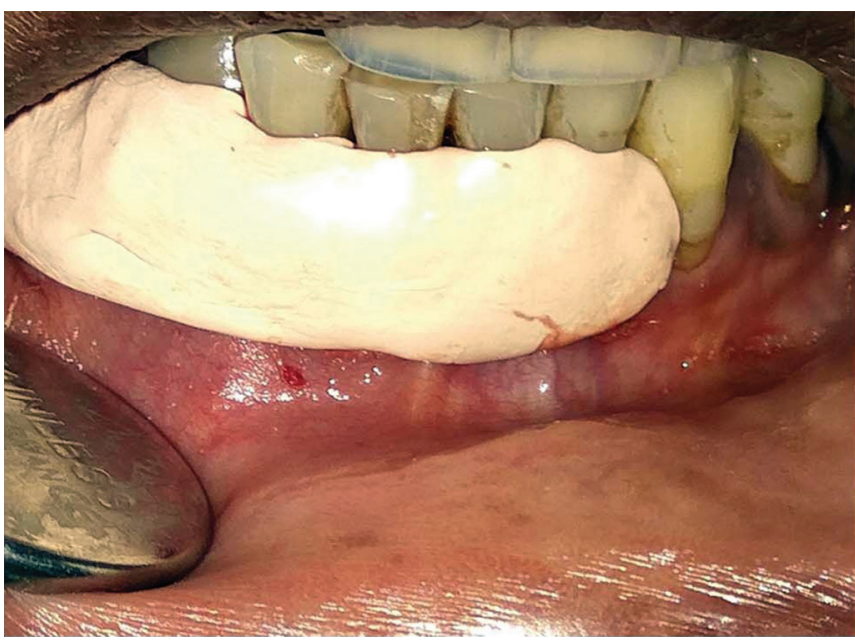

Fig. 5: Periodontal Coe-Pak applied

displacement. The exposed root surfaces were scaled and root planed. Following this, the cervical step at the CEJ was eliminated using an aerator and a diamond bur.

Preparation of PRF Membrane After the recipient site preparation was completed, $5 \mathrm{~mL}$ of venous blood was drawn in test tubes without an anticoagulant, and centrifuged immediately. It was centrifuged for 12 minutes at $2,700 \mathrm{rpm}$. The resultant product consisted of the following three layers: The topmost layer consisted of acellular platelet-poor plasma, a PRF clot in the middle, and red blood cells (RBCs) at the bottom. After centrifugation, the PRF clot was obtained, separated from the RBC base using scissors, and placed in a sterile appen dish. The PRF membrane was prepared by placing it into a petri dish. At the recipient site, the PRF clot was placed over the denuded root surfaces (Fig. 3). The flap was coronally advanced to cover the membrane as well as the defect and sutured (Fig. 4). A tin foil and periodontal dressing were placed over the surgical area (Fig. 5).

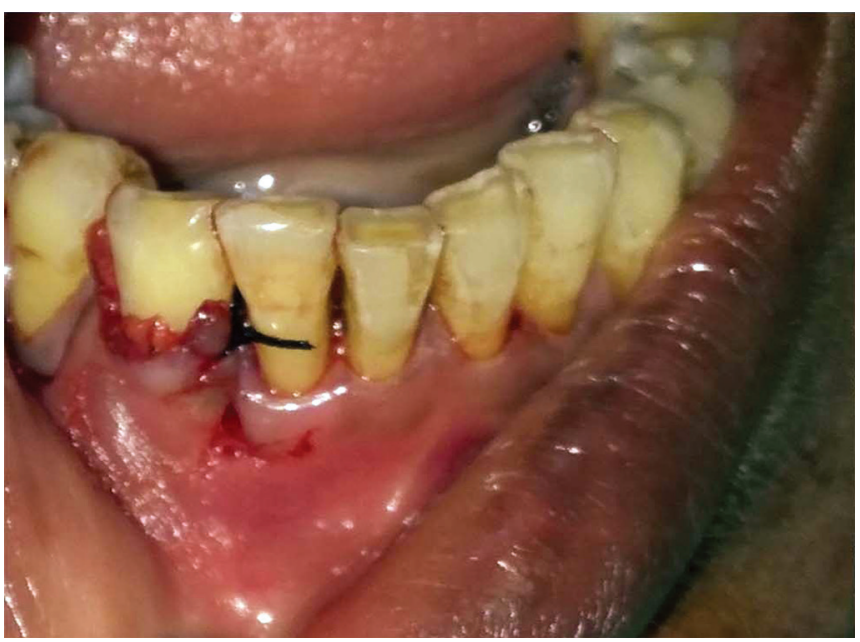

Fig. 4: Flap was coronally advanced and suture overlying the PRF

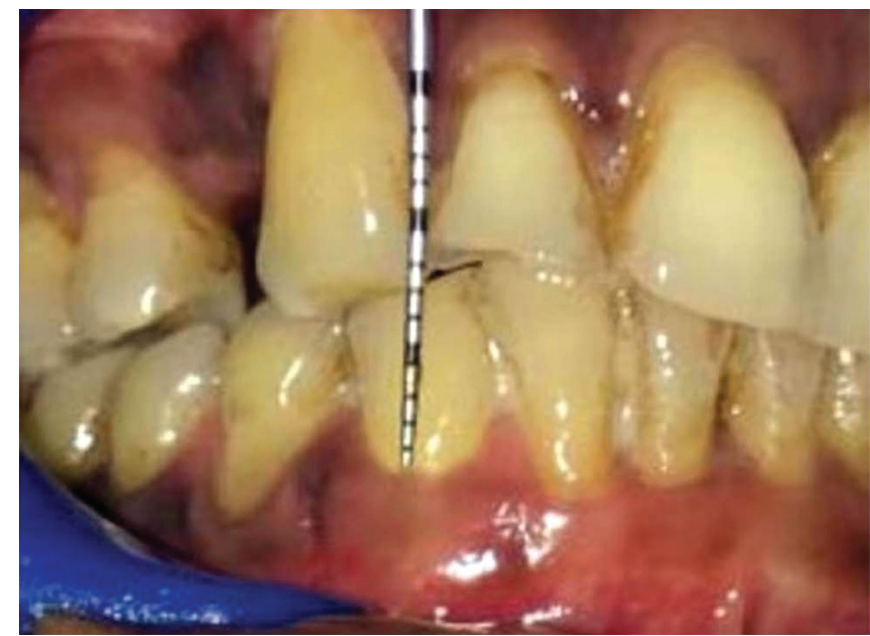

Fig. 6: Six-month postoperative period showing predictable root coverage

Postoperative Care The patient was advised to use $0.2 \%$ chlorhexidine digluconate mouth rinse, twice daily. Systemic analgesics were prescribed, and he was advised to follow the routine postoperative instructions. The dressing and sutures were removed 10 days after surgery, and patients were followed up for 6 months (Fig. 6).

\section{Coronally Advanced Flap with Amniotic Membrane Site 2}

A similar surgical procedure was followed to 23 (Figs 7 to 9) to that for the PRF membrane. The commercially available amniotic membranes were cut into the desired shape and length with scissors and placed on the recession site (Fig. 10) and were stabilized by resorable suture (Fig. 11) with flap, which was coronally advanced and sutured (Fig. 12). A tin foil and periodontal dressing were placed over the surgical area (Fig. 13). Patient satisfaction was recorded after 6 months (Fig. 14) with a questionnaire. The result of the procedures relative to 


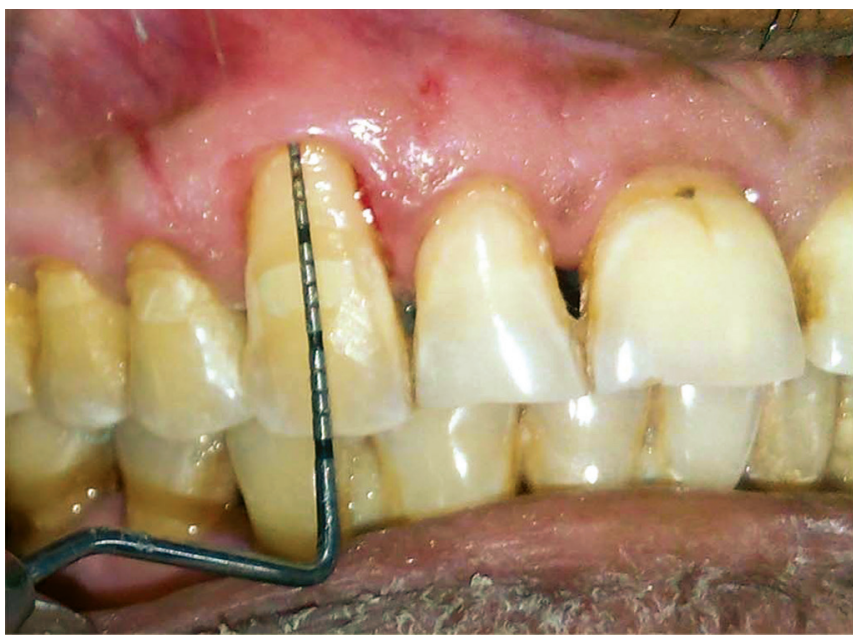

Fig. 7: Miller class II gingival recession in the upper right canine

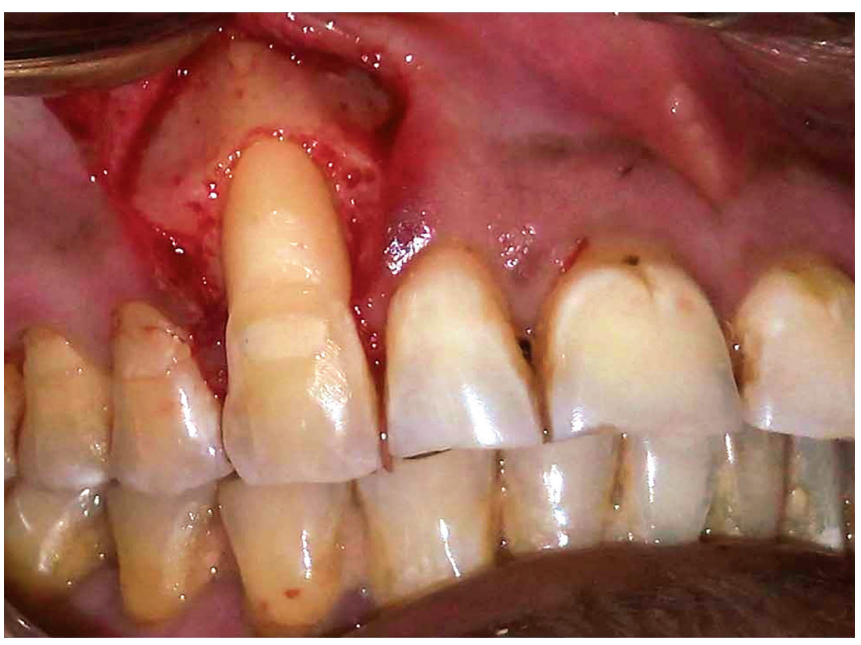

Fig. 9: Elevation of periosteal flap

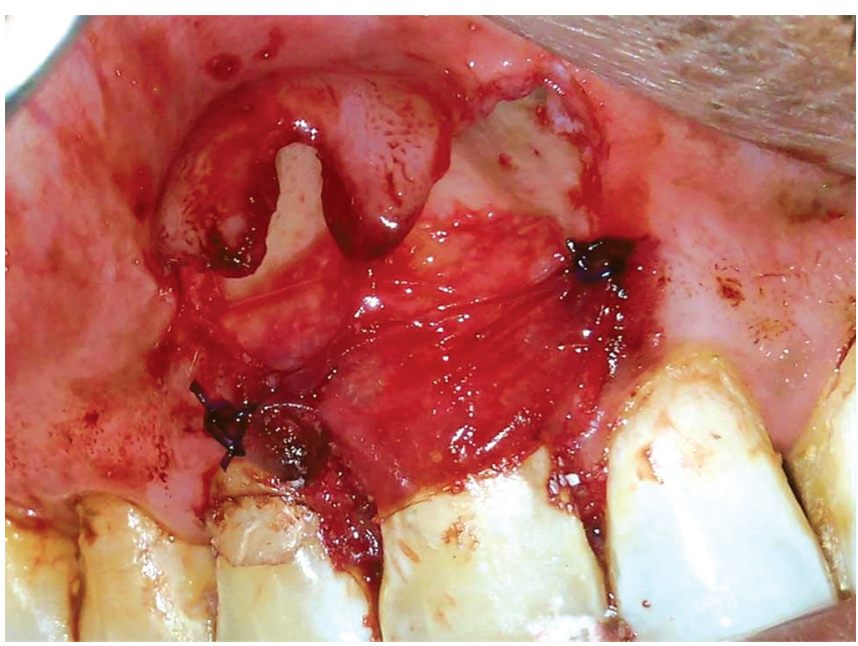

Fig. 11: Stabilization of amniotic membrane with restorable suture

esthetics, root sensitivity (before and after surgery), and the postoperative period in terms of esthetic outcome patients expressed their opinion of each treated tooth by selecting one of the following choices: Bad, sufficient,

\begin{tabular}{|l|}
\hline $\begin{array}{c}\text { TATA MEMORIAL HOSPITAL TISSUE BANK } \\
\text { Dr. Ernest Borges Road, Parel, Mumbai - } 400012 . \\
\text { Phone : } 2417 \text { 7000 Extn. : } 4122 / 4112\end{array}$ \\
\hline Graft No. : \\
Size : $\frac{A 767 \mathrm{~B}-15}{7 \times 359-\mathrm{cm}}$ \\
Radiation Batch No. $: \frac{4 / 15}{2018}$ \\
Expiry Date : \\
\hline STORAGE AT ROOM TEMPERATURE \\
\hline FREEZE-DRIED, IRRADIATED AMNION \\
\hline
\end{tabular}

Fig. 8: Human amniotic membrane

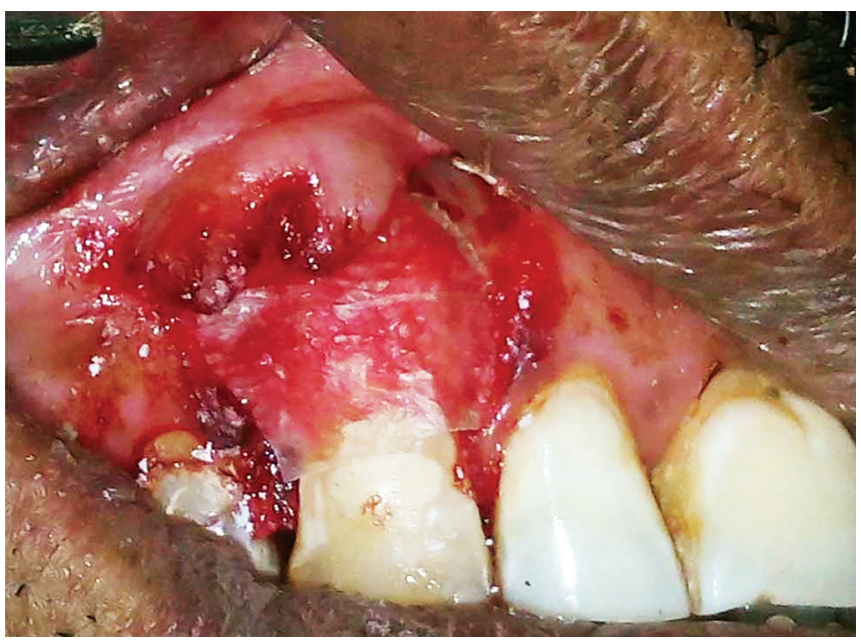

Fig. 10: Adaptation of amniotic membrane over the exposed root surface

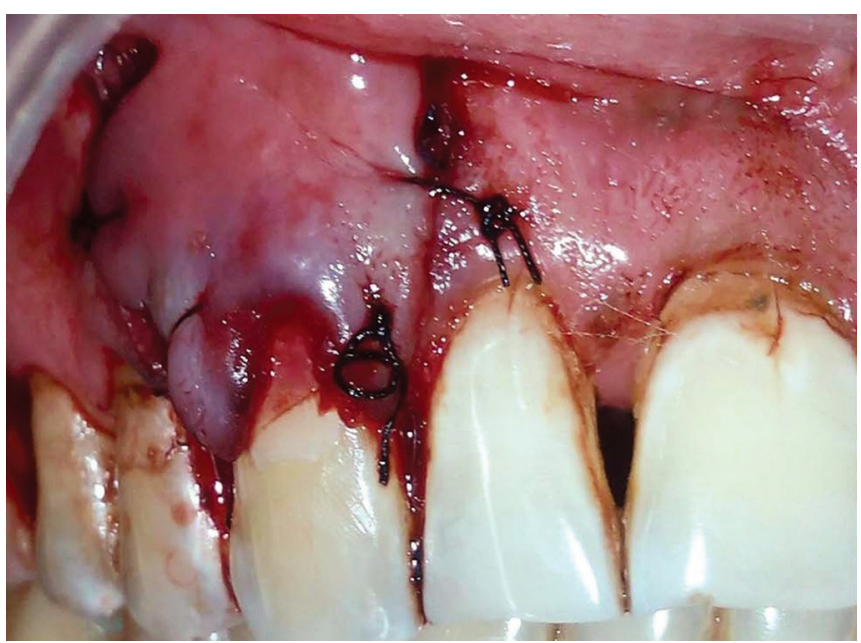

Fig. 12: Flap was coronally advanced and suture overlying the amniotic membrane

good, or excellent. In terms of the pain experienced in the postoperative period, patients selected one of the following choices: None, low, moderate, or severe. Patients were also asked to indicate if one technique was preferable 


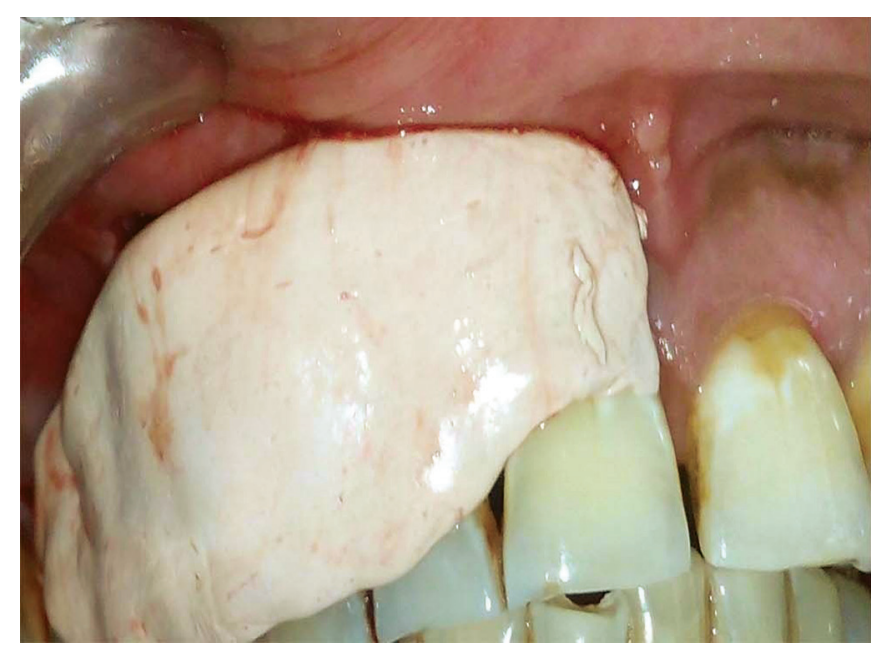

Fig. 13: Periodontal Coe-Pak applied

to the other in terms of the postoperative period and esthetics. The patients had been instructed to mark when (date and hour) the analgesic medication was taken. The subject's overall postoperative pain was also assessed using a $100-\mathrm{mm}$ horizontal visual analog scale, with the left endpoint marking no pain and the right endpoint marking extreme pain.

\section{Statistical Analysis}

Descriptive statistics were expressed as mean \pm standard deviation. Repeated measure analysis of variance was used for examination of differences within and between treatments regarding the PD, CAL, and WKT parameters. The nonparametric Wilcoxon test was used to detect differences within and between groups regarding the percentage of RC, TKT, RH, and RW parameters. The Pearson correlation coefficient was used to determine possible correlation between TKT at base line and RH reduction. The Wilcoxon test was applied to compare the pain intensity and the number of pills used in the postoperative period. A significance level of 0.05 was employed in all statistical comparisons.

\section{RESULTS}

Healing was uneventful for all 15 patients, and no patients were excluded from the study. The recessions were located in maxillary and mandibular canines. Full-mouth sulcus bleeding index and visible plaque index were maintained below $20 \%$, indicating a good standard of supragingival plaque control during the study period. No statistically significant differences were observed between groups in any of the clinical parameters at baseline. In the CAF with PRF group, statistically significant changes from baseline were found for RH, RW, WKT, and CAL. The $\mathrm{RH}$ decreased from $2.10-0.58 \mathrm{~mm}$ to $0.23-0.27 \mathrm{~mm}$,

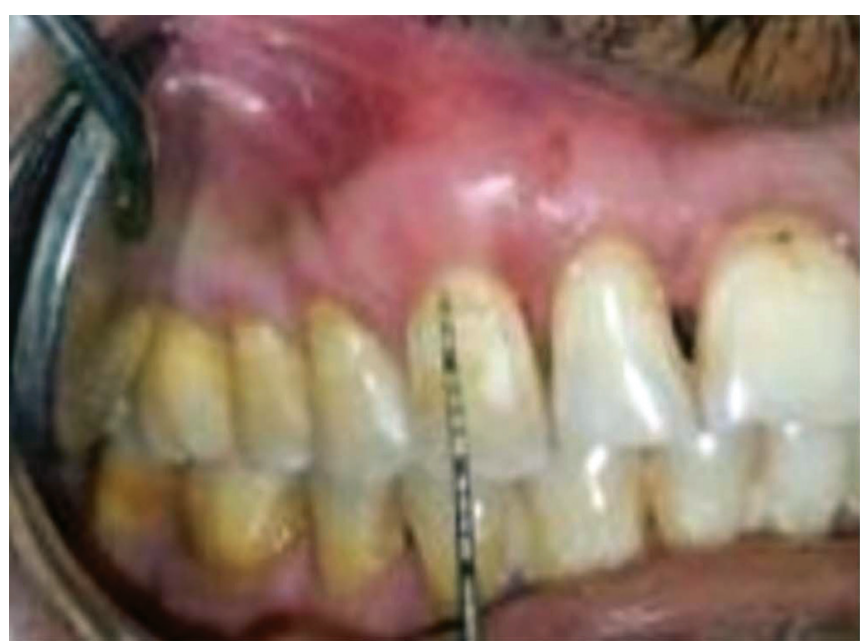

Fig. 14: Six months postoperative period showing predictable root coverage

corresponding to a mean root coverage of $92.95 \%$ to $11.46 \%$. In the SCPF with amniotic membrane group, statistically significant changes from baseline were found for all parameters, except PD. The RH decreased from $2.17-0.61 \mathrm{~mm}$ to $0.12-0.21 \mathrm{~mm}$, corresponding to a mean root coverage of $97.40 \%$ to $8.69 \%$. A statistically significant difference favoring the CAF with amniotic membrane group was observed in the increase of TKT. Complete root coverage was accomplished in 56.94\% (9 out of 15) of the treated cases in the CAF with PRF group, and in $76.47 \%$ (13 out of 15 ) in the CAF with amniotic membrane group. There was no significant correlation between TKT at baseline and the amount of root coverage at 6 months for either technique. The esthetic outcome was as follows: In the CAF with PRF group, 9 subjects out of 15 reported an excellent esthetic result, 5 indicated a good result, and 1 reported a sufficient result, while in the CAF with amniotic membrane group, 12 subjects reported an excellent result and the 3 indicate a good result. When asked about the preferred procedure, the two treatments demonstrated essentially equal results regardless to postoperative period; however, no patients in the CAF with PRF group and CAF with amniotic membranes were merged.

\section{DISCUSSION}

The PRF is a second-generation platelet concentrate and is defined as an autologous L-PRF biomaterial, which was first developed by Choukroun et al. ${ }^{13}$ It has been used extensively in combination with bone graft materials for periodontal regeneration, ridge augmentation, sinus lift procedures for implant placement, and for coverage of recession defects in the form of a membrane. This membrane consists of a fibrin 3D polymerized matrix in a specific structure, with the incorporation of platelets, leukocytes, growth factors, and the presence of circulating 
stem cells. The amniotic membrane is a composite membrane consisting of a pluripotent cellular element embedded in a semipermeable membranous structure. ${ }^{14}$ It has been shown that the amniotic membrane is an immune-tolerant structure. Meanwhile, the existence of pluripotent stem cells possessing the ability of transdifferentiation to other cellular elements of the periodontium makes it a suitable candidate for guided tissue regeneration (GTR). Excellent revascularization of the amniotic membrane is another favorable property of this natural structure. The clinical application of the amniotic membrane for GTR, while fulfilling the current mechanical concept of GTR, amends it with the modern concept of biological GTR. The biomechanical GTR proposed herein, using the amniotic membrane, not only maintains the structural and anatomical configuration of the regenerated tissues, but also contributes to the enhancement of healing through reduction of postoperative scarring and subsequent loss of function, and also provides a rich source of stem cells. It has been demonstrated that the amniotic membrane enhances the gingival wound healing properties and reduces scarring.

The human amniotic membrane (HAM) has been used in the field of oral and maxillofacial surgery from 1969 onward, because of its immunological preference and its pain-reducing, antimicrobial, mechanical, and side-dependent adhesive or antiadhesive properties. The effects of HAM on dermal and mucosal re-epithelialization have been highlighted. Typically, HAM is applied after being banked in a glycerol-preserved, dimethyl sulfoxide -preserved or freeze-dried and irradiated state. Even as the use of HAM in flap surgery and in intraoral and extraoral lining is reported frequently, novel HAM applications in posttraumatic orbital surgery and temporomandibular joint surgery have been added since 2010. Tissue engineering with HAM is a fast expanding field with a high variety of future options. ${ }^{15}$ The other indications for the use of amniotic membrane are in the field of oral surgery, wherein the membrane transplantation shows rapid epithelialization in both granulation tissue and collagen formation, but which suppresses inflammation, suggesting that amniotic membrane transplantation may promote rapid gingival wound healing compared with secondary healing, and has been seen in rabbits. ${ }^{16}$ Moreover, when used in vestibuloplasty, these grafts of the amniotic membrane are viable and reliable for covering of the raw surface, as they prevent secondary contraction after vestibuloplasty and maintain the postoperative vestibular depth. ${ }^{17}$ An average gain of 4 to $6 \mathrm{~mm}$ in the depth of the labial vestibule has been noted, proving that the amniotic membrane can be a favorable graft material for vestibuloplasty, promoting healing and preventing relapse. It is easily available and preserved and is a cost-effective material. ${ }^{18}$
The amniotic membrane, a novel preservable material derived from the human amnion, has been introduced clinically in ophthalmology and other fields. This membrane is available as a wound dressing material for surgical wounds of the tongue and buccal mucosa, but has not been used on wounds of the alveolar mucosa. This article has reported two cases in which intraoral alveolar wounds with bone exposure have been successfully treated with the use of amniotic membrane. The cases are of a 74-year-old woman with gingival leukoplakia of the edentulous mandible and a 43-year-old man, who underwent vestibuloplasty of the reconstructed mandible. The results indicate that the amniotic membrane is a useful dressing material not only for soft tissue wounds, but also for exposed bone in the oral cavity. ${ }^{19}$ Oro nasal fistulas are a frequent complication after cleft palate surgery. Use of the amniotic membrane has been successful for oro nasal fistula repair and use of the multilayer technique and protective plate utilization prevent membrane ruptures. ${ }^{20}$ A recent 6-month study evaluated the use of PRF in the treatment of multiple gingival recessions with CAF procedure, and found significant improvement during the early periodontal healing phase, with a thick and stable final remodeled gingiva. However, another randomized clinical trial in the same year reported an inferior root coverage of about $80.7 \%$ at the test site (CAF + PRF) as compared with about $91.5 \%$ achieved at the control site (CAF), but it was an additional gain in gingival/mucosal thickness compared with conventional therapy. ${ }^{21}$ An increase in thickness of the keratinized tissues, reported in both studies, might contribute to a long-term stable clinical outcome, with reduced probability of the recurrence of recession. ${ }^{22}$

The objective of this split-mouth, randomized, single blind, and controlled clinical trial was to compare the CAF with PRF procedure with the CAF with amniotic membrane procedure for the treatment of buccal gingival recessions. Considering the study design and the groups' homogeneity at baseline, differences in clinical outcomes can be attributed to the treatments employed. Present data indicate that the two surgical approaches adopted in the study were highly effective and predictable in obtaining root coverage of gingival recessions and esthetic improvements. In fact, both techniques resulted in a very high percentage of root coverage $(92.95 \%$ in the CAF with PRF group and $97.40 \%$ in the CAF with amniotic membrane group), and complete soft tissue root coverage was achieved in the majority of treated cases (56.94\% in the CAF with PRF group and $76.47 \%$ in the CAF with amniotic membrane group). These results are in agreement with studies using various flap and graft techniques in controlled clinical trials. However, the comparison between studies is compromised by the different methods used to measure the clinical parameters. The 
measurement techniques that have a resolution of 1.0 or $0.5 \mathrm{~mm}$ tend to provide more favorable results than those that have a resolution of $0.1 \mathrm{~mm}$. For example, the residual recessions (0.26-0.62 $\mathrm{mm}$ ) observed in the present study probably would not be quantified if the caliper was not used. There is a need to investigate root coverage procedures as they relate to patient-oriented outcomes, such as esthetics, root sensitivity, and postoperative morbidities. ${ }^{4}$ The CAF with PRF and CAF with amniotic membrane showed an esthetic improvement after 6 months, and patients were generally satisfied with both procedures (96.1 and 100\% respectively). There is no statistical difference between pain intensity.

\section{CONCLUSION}

The present study demonstrated that both CAF with PRF and CAF with amniotic membrane were effective in providing root coverage in Miller class I gingival recession defects where the patient presented with at least $2 \mathrm{~mm}$ of keratinized gingiva prior to the root coverage surgical procedure, However, CAF with amniotic membrane gives better results.

\section{REFERENCES}

1. Wennström JL, Zucchelli G. Increased gingival dimensions. A significant factor for successful outcome of root coverage procedures? J Clin Periodontol 1996 Aug 1;23(8):770-777.

2. Löe H, Ånerud $\AA$, Boysen $H$. The natural history of periodontal disease in man: prevalence, severity, and extent of gingival recession. J Periodontol 1992 Jun;63(6):489-495.

3. Clauser C, Nieri M, Franceschi D, Pagliaro U, Pini-Prato G. Evidence-based mucogingival therapy. Part 2: Ordinary and individual patient data meta-analyses of surgical treatment of recession using complete root coverage as the outcome variable. J Periodontol 2003 May 1;74(5):741-756.

4. Oates TW, Robinson M, Gunsolley JC. Surgical therapies for the treatment of gingival recession. A systematic review. Ann Periodontol 2003 Dec 1;8(1):303-320.

5. Choukroun J, Diss A, Simonperi A, Girard MO, Schoeffler C, Dohan SL, Dohan AJJ, Mouhyi J, Dohan DM. Platelet-rich fibrin (PRF): a second generation platelet concentrates. Part V: histologic evaluation of PRF effects on bone allograft maturation in sinus lift. Oral Surg Oral Med Med Oral Pathol Oral Radiol Endod 2006 Mar;101(3):299-303.

6. Diss A, Dohan DM, Mouhyi J, Mahler P. Osteotome sinus floor elevation using Choukroun's platelet-rich fibrin as grafting material: a 1-year prospective pilot study with microthreaded implants. Oral Surg Oral Med Oral Pathol Oral Radiol Endod 2008 May 31;105(5):572-579.

7. Simonpiere A, Del Corso M, Samnartino G, Dohan Ehrenfest DM. The relevance of Choukroun's Platelet-rich fibrin and metronidazole during complex maxillary rehabilitation using bone allograft. Part II: implant surgery, prosthodontic and survival. Implant Dent 2009 Jun;18(3):220-229.
8. Ilancheran S, Moodley Y, Manuelpillai U. Human fetal membranes: a source of stem cells for tissue regeneration and repair? Placenta 2009 Jan 31;30(1):2-10.

9. Davis JS. Skin transplantation with a review of 550 cases at the Johns Hopkins Hospital Reports. Johns Hopkins Med J 1910;15:307-396.

10. Niknejad H, Peirovi H, Jorjani M, Ahmadiani A, Ghanavi J, Seifalian AM. Properties of the amniotic membrane for potential use in tissue engineering. Eur Cells Mater 2008 Apr;15:88-99.

11. Velez I, Parker WB, Siegel MA, Hernandez M. Cryopreserved amniotic membrane for modulation of periodontal soft tissue healing: a pilot study. J Periodontol 2010 Dec;81(12):1797-1804.

12. Gurinsky B. A novel dehydrated amnion allograft for use in the treatment of gingival recession: an observational case series. JIACD 2009 Mar;1:124-130.

13. Dohan DM, Choukroun J, Diss A, Dohan SL, Dohan AJ, Mouhyi J, Gogly B. Platelet-rich fibrin (PRF): a secondgeneration platelet concentrate. Part II: platelet-related biologic features. Oral Sur Oral Med Oral Pathol Oral Radiol Endodontol 2006 Mar 31;101(3):e45-e50.

14. Adams EA, Choi HM, Cheung CY, Brace RA. Comparison of amniotic and intramembranous unidirectional permeabilities in late-gestation sheep. Am J Obstet Gynecol 2005 Jul 31;193(1):247-255.

15. Kesting MR, Wolff KD, Nobis CP, Rohleder NH. Amniotic membrane in oral and maxillofacial surgery. Oral Maxillofac Surg 2012 Dec;18(2):153-164.

16. Rinastiti M, Santoso AL, Sosroseno W. Histological evaluation of rabbit gingival wound healing transplanted with human amniotic membrane. Int J Oral Maxillofac Surg 2006 Mar 31;35(3):247-251.

17. Kothari CR, Goudar G, Hallur N, Sikkerimath B, Gudi S, Kothari MC. Use of amnion as a graft material in vestibuloplasty: a clinical study. Br J Oral Maxillofac Surg 2012 Sep 30;50(6):545-549.

18. Sharma Y, Maria A, Kaur P. Effectiveness of human amnion as a graft material in lower anterior ridge vestibuloplasty: a clinical study. J Maxillofac Oral Surg 2011 Dec 1;10(4): 283-287.

19. Tsuno H, Arai N, Sakai C, Okabe M, Koike C, Yoshida T, Nikaido T, Noguchi M. Intraoral application of hyperdry amniotic membrane to surgically exposed bone surface. Oral Surg Oral Med Oral Pathol Oral Radiol 2014 Feb 28;117(2): e83-e87.

20. Rohleder NH, Loeffelbein DJ, Feistl W, Eddicks M, Wolff KD, Gulati A, Steinstraesser L, Kesting MR. Repair of oronasal fistulae by interposition of multilayered amniotic membrane allograft. Plast Reconstr Surg 2013 Jul 1;132(1):172-181.

21. Del Corso M, Sammartino G, Dohan Ehrenfest DM. Clinical evaluation of a modified coronally advanced flap alone or in combination with a platelet-rich fibrin membrane for the treatment of adjacent multiple gingival recessions: a 6 month study. J Periodontol 2009 Nov;80(11):1694-1697.

22. Aroca S, Keglevich T, Barbieri B, Gera I, Etienne D. Clinical evaluation of a modified coronally advanced flap alone or in combination with a platelet-rich fibrin membrane for the treatment of adjacent multiple gingival recessions: a 6-month study. J Periodontol 2009 Feb;80(2):244-252. 